\title{
Adipose-derived Mesenchymal Stem Cells Protect Neurons by Inhibiting Microglial Inflammation
}

Ke Yan ( $\sim$ yan2008ke@126.com )

Yangzhou University Medical Academy https://orcid.org/0000-0002-1534-0011

Jia-rong Bian

Yangzhou University Medical Academy

Liang He

Yangzhou University Medical Academy

Bing-wei Song

Yangzhou University Medical Academy

Lun Dong

Yangzhou University Medical Academy

Jia-wei He

Yangzhou University Medical Academy

Lin-hai Shen

Yangzhou University Medical Academy

Xin-zhi Zhou

Yangzhou University Medical Academy

Yong Zhen

Yangzhou University Medical Academy

\section{Research Article}

Keywords: Adipose-derived mesenchymal stem cells, central nervous system, immunosuppression, microglia activation, neuroinflammation

Posted Date: February 15th, 2021

DOl: https://doi.org/10.21203/rs.3.rs-226928/v1

License: (c) (1) This work is licensed under a Creative Commons Attribution 4.0 International License. Read Full License 


\section{Abstract}

Microglia are considered neurotoxic when activated. Regulating microglial activity during inflammatory stress is an important aspect in treating many central nervous system (CNS) diseases because microglia produce various substances that affect neurons and glial cells. Adipose-derived mesenchymal stem cells (ADSCs) have been shown to have a therapeutic effect following neuronal damage, which is attributed to the fact that ADSCs can differentiate into neurons or produce neurotrophic factors. However, whether ADSCs play a therapeutic role by affecting microglial activity is unclear. In this study, microglia were stimulated with lipopolysaccharide (LPS) to trigger an inflammatory response, and these activated microglia were co-cultured either directly with ADSCs or in ADSCs-conditioned media (ADSCs-CM). ADSCs or ADSCs-CM significantly inhibited not only microglia migration and phagocytosis but also the secretion of proinflammatory factors by microglia. Furthermore, primary hippocampal neurons were cultured with activated microglia in a Transwell co-culture system, and ADSCs or ADSCs-CM were added to investigate whether ADSCs could influence neuronal apoptosis. ADSCs could significantly inhibit neuronal apoptosis in the inflammatory state. The experimental results provided evidence that ADSCs might control microglial activation by producing different substances, indicating that ADSCs can be effective therapeutic strategies for treating diseases associated with microglial inflammation.

\section{Introduction}

Microglia are innate immune cells located in the brain parenchyma of the central nervous system (CNS) behind the blood-brain barrier (BBB) [1]. They are part of the mononuclear phagocyte lineage and correlated with other organ-specific macrophage populations, such as osteoclasts of the bone and Kupffer cells of the liver. Under physiological conditions, microglia is present with a ramified appearance, called "resting" state. In this state, they express low levels of many immune receptors, including Toll-like receptors, NOD-like receptors, and major histocompatibility complex class II molecules, which are essential for the initiation and propagation of immune responses [2]. Certain stimuli such as ischemic damage or oxidative stress can transform microglia from a resting state to an activated state. Activated microglia release many proinflammatory cytokines such as tumor necrosis factor-a (TNF-a), interleukin$1 \beta$ (IL-1 $\beta$ ), and nitric oxide (NO), which are toxic to neighboring neurons [3-5]. Furthermore, the phagocytosis of activated microglia is significantly enhanced. In this state, they can phagocytose not only dead cells and cell debris but also normal neurons [6]. Experiments have also shown that continuous microglial activation induces the recruitment of peripheral cells into the CNS, leading to chronic neuroinflammation [7, 8]. Excessive microglial activation may lead to the pathophysiology of several disorders such as ischemic stroke, traumatic brain injury (TBI), and neurodegenerative diseases including Parkinson's, Alzheimer's, and Huntington's diseases [9-12].

Adipose-derived mesenchymal stem cells (ADSCs) are considered excellent candidates for cell-based tissue engineering. ADSCs are less sensitive to autograft immune rejection because of their lack of immunogenicity [13]. Under specific conditions, ADSCs can differentiate along different lineages, such as osteoblasts, chondrocytes, adipocytes, myocytes, and embryonic lineages [14]. The role of ADSCs as 
alternative therapeutic cells has been demonstrated by multiple experiments. For example, human ADSCs have been used successfully to treat physiological and pathological vulvar dystrophies [15]. When ADSCs are transplanted into the heart of a patient with myocardial infarction, they promote the regeneration of heart cells [16]. In an in vivo model of Kaposi's sarcoma, the intravenous injection of human ADSCs resulted in the homing of ADSCs to the tumor site and subsequent suppression of tumor growth [17]. ADSCs are also important therapeutically in the CNS of animal models of TBI and spinal cord injury (SCI) $[18,19]$. The explanation for this phenomenon may be the transdifferentiation of ADSCs, which induces them to replace injured neurons [20]. However, ADSCs can synthesize and secrete both growth and neurotrophic elements, such as nerve growth factor, brain-derived neurotrophic factor, vascular endothelial growth factor, and hepatocyte growth factor (HGF), all of which are crucial components for neural damage repair [21-25]. In recent years, a large number of studies have found that ADSCs can slow down the pathological process of neurodegenerative diseases and promote neurological function recovery. For example, Azim et al. demonstrated that the transplantation of homologous ADSCs remyelinated demyelinated corpus callosum axons after intravenous transplantation in a cuprizone mouse model of multiple sclerosis [26]. ADSCs transplantation also inhibited pro-inflammatory responses, enhanced anti-inflammatory pathways, alleviated A $\beta$ pathology in the brains of APP/PS1 transgenic $A D$ model mice, and mitigated the cognitive decline in model animals [27]. The results of these preliminary studies suggested that the treatment of neurodegenerative diseases by ADCs might be related to their immunomodulatory abilities.

In this study, rat ADSCs were used in combination with rat microglia to investigate whether ADSCs could affect microglia activity. Additionally, primary hippocampal neurons were cultured with activated microglia in a Transwell co-culture system, and ADSCs or ADSCs-CM were added to the co-culture system, to investigate whether ADSCs could inhibit neuronal apoptosis by inhibiting neuroinflammation caused by microglial activation.

\section{Materials And Methods}

\section{Animals}

Sprague-Dawley (SD) rats were obtained from the Laboratory Animal Center of Yangzhou University (Yangzhou, China). All animal experiments were conducted in accordance with the National Institutes of Health Guide for the Care and Use of Laboratory Animals. All experimental procedures were approved by the Yangzhou University Ethics Committee.

\section{Isolation of ADSCs and collection of ADSCs-CM}

Rat adipose tissues were first minced with a pair of microsurgical scissors and digested with $0.075 \%$ collagenase at $37^{\circ} \mathrm{C}$ for $15 \mathrm{~min}$. The enzyme activity was then neutralized with Dulbecco's Modified Eagle's Medium (DMEM, Gibco, NY, USA), containing $1 \%$ fetal bovine serum (FBS, Gibco), followed by centrifugation at $300 \times g$ for 10 min to obtain a pellet. The pellet was resuspended in DMEM containing $1 \%$ FBS and penicillin/streptomycin to obtain mononuclear cells. The mononuclear cells were cultured at 
$37^{\circ} \mathrm{C}$ in a $5 \% \mathrm{CO}_{2}$ humidified atmosphere for 2 days, after which the supernatant was replaced with fresh media to remove nonadherent cells. The remaining adherent cells (primary ADSCs) were passaged every 2 days. Cells from passages three through five were used in the experiments. The expression of the typical markers CD44 and CD90 and the absence of the hematopoietic markers CD34 and CD45 were assessed by cytofluorometric analysis.

To isolate ADSCs-CM, ADSCs were cultured in $25-\mathrm{cm}^{2}$ flasks in $5 \mathrm{~mL}$ of DMEM with $1 \%$ FBS. After $24 \mathrm{~h}$, the supernatant was replaced with $5 \mathrm{~mL}$ of fresh DMEM without FBS and the culturing was continued for $24 \mathrm{~h}$. Then, the culture medium was collected and centrifuged at 1,000 rpm for $5 \mathrm{~min}$ to remove debris. The obtained media was defined as ADSCs-CM in this study.

\section{Microglia culture}

Primary microglia were enriched by the method described by Giulian and Baker [28]. Briefly, 2-day-old SD rats were sacrificed, and the cerebral hemispheres were dissected out. The meninges were peeled off, and the hippocampus, basal ganglion, and olfactory bulbs were removed with microsurgical instruments. The remaining cortices were then minced with microsurgical scissors and incubated in $0.25 \%$ trypsin (Gibco) at $37^{\circ} \mathrm{C}$ for $15 \mathrm{~min}$. Enzyme-digested dissociated cells were neutralized with $1 \mathrm{~mL}$ of FBS, followed by washing and centrifugation ( $300 \times \mathrm{g}$ for $10 \mathrm{~min}$ ). The obtained pellet was resuspended and plated into $75-\mathrm{cm}^{2}$ flasks with $15 \mathrm{~mL}$ of DMEM containing $1 \%$ FBS. The culture media was replaced with $15 \mathrm{~mL}$ of fresh growth media $24 \mathrm{~h}$ later. The mixed brain cell cultures were then incubated for an additional 4 days. At the end of this stage, mixed brain cells were stratified. The round, bright cells in the upper layer were microglia. The culture flasks were put into a reciprocating shaker bath to oscillate for $5 \mathrm{~min}$; the microglia could be easily detached from the mixed cells into the supernatant. The supernatant was collected and centrifuged at $300 \times g$ for $10 \mathrm{~min}$. The precipitates obtained (mainly microglia) were resuspended and plated into $75-\mathrm{cm}^{2}$ flasks coated with poly-L-lysine (Sigma-Aldrich, St. Louis, MO, USA) for further use. The expression of the typical marker CD11b was assessed by cytofluorometric analysis.

\section{Hippocampal neuron culture}

Primary hippocampal neural cells were obtained from 18-day-old SD rat embryos of both sexes. The hippocampus was dissected and collected in Hank's solution, followed by trypsinization $(0.125 \%)$ for 15 min in a $37^{\circ} \mathrm{C}$ water bath. The cells were collected by centrifugation $(100 \times g, 10 \mathrm{~min})$ and resuspended in neurobasal culture media containing $2 \%$ B27 supplement, $25 \mathrm{mM}$ glutamate, and $50 \mathrm{U} / \mathrm{mL}$ penicillinstreptomycin (all from Gibco). The cells were then plated in 24-well plates coated with $50 \mu \mathrm{g} / \mathrm{mL}$ poly-Dlysine (Sigma-Aldrich) at a density of $2 \times 10^{5} \mathrm{cells} / \mathrm{mL}(0.5 \mathrm{~mL} /$ well), or in $35-\mathrm{mm}$ dishes $(2 \mathrm{~mL} / \mathrm{dish})$. Neuron cultures were incubated at $37^{\circ} \mathrm{C}$ in a humidified $5 \% \mathrm{CO}_{2}$ atmosphere. Half of the culture media was substituted with fresh neurobasal culture media plus 2\% B27 supplements every 3 days.

\section{Co-culture in the Transwell system}

The neuroprotective effect of ADSCs on neurons was explored by exposing the co-cultures of neurons and microglia to ADSCs-CM in a Transwell system with a $0.4-\mu \mathrm{m}$ pore size (Corning, Corning, NY, USA). 
Neurons $\left(1 \times 10^{5}\right.$ cells) were seeded into the bottom well of a 6 -well Transwell system, while microglial cells $\left(2 \times 10^{5}\right.$ cells) were cultured in the upper chamber. The cells were first maintained in a neurobasal culture medium supplemented with $1 \%$ FBS for $24 \mathrm{~h}$. Afterward, the medium was replaced with the same volume of neurobasal culture medium with or without $1 \mu \mathrm{g} / \mathrm{mL}$ lipopolysaccharide (LPS), and the cells were incubated for 12,24 , and $48 \mathrm{~h}$. Transwell cellular co-cultures were supplied with ADSCs-CM with or without $1 \mu \mathrm{g} / \mathrm{mL}$ LPS for the same time points to analyze the role of ADSCs in microglial inflammation. Each sample was cultured in duplicate, and each co-culture experiment was repeated at least three times.

\section{Neuronal apoptosis assays}

The TUNEL assay was performed using a commercial kit (In Situ Cell Death Detection Kit; Roche, IN, USA) following the manufacturer's protocols. Briefly, freshly isolated hippocampal neurons were incubated in the bottom well for $24 \mathrm{~h}$ in the aforementioned co-culture system. The medium was then changed into neurobasal culture medium and ADSCs-CM with or without $1 \mu \mathrm{g} / \mathrm{mL}$ LPS, and the cells were incubated for 12,24 , and $48 \mathrm{~h}$. The obtained neurons were fixed with $4 \%$ paraformaldehyde for $20 \mathrm{~min}$, followed by permeabilization with $0.1 \%$ Triton $\mathrm{X}-100$ for $10 \mathrm{~min}$ at room temperature. Then, the neurons were incubated with TUNEL reaction mixture for $60 \mathrm{~min}$ in a $37^{\circ} \mathrm{C}$ humidified chamber. Finally, the cells were washed with phosphate-buffered saline (PBS) and cultured with 4囚,6-diamidino-2-phenylindole (DAPI; Sigma-Aldrich) for $10 \mathrm{~min}$. Apoptotic cells were counted under $400 \times$ magnification by independent observers using imaging software (Image-Pro Plus, Media Cybernetics, MD, USA).

\section{Treatment of microglial cells}

Microglia were divided into four groups to study the effect of ADSCs on microglial secretion, migration, phagocytic capacity, and phenotypic expression.

1) Control group: microglia were incubated in DMEM containing $1 \%$ FBS.

2) LPS group: microglia were incubated in DMEM containing $1 \%$ FBS and $1 \mu \mathrm{g} / \mathrm{mL}$ LPS.

3) ADSCs-CM group: microglia were incubated in ADSCs-CM containing 1\% FBS.

4) $\mathrm{CM}+$ LPS group: microglia were incubated in ADSCs-CM containing $1 \% \mathrm{FBS}$ and $1 \mu \mathrm{g} / \mathrm{mL}$ LPS.

\section{Cytokine and chemokine measurements}

Bio-Plex Pro assay (Bio-Rad Laboratories, CA, USA) was used to measure the levels of cytokines and chemokines secreted by microglia. Briefly, $2 \times 10^{5}$ microglial cells were plated into 6-well plates and incubated in DMEM containing 1\% FBS. After $24 \mathrm{~h}$, the media was replaced with DMEM or ADSCs-CM, and the cells were incubated for an additional $24 \mathrm{~h}$ with or without LPS $(1 \mu \mathrm{g} / \mathrm{mL})$. The supernatant was collected and centrifuged at $300 \times g$ to remove impurities, and the levels of six cytokines/chemokines (TNF-a, IL-1 $\beta$, IL-10, MCP-1, RANTES, and MIP-2) were measured using the Bio-Plex Pro assay kit following the manufacturer's protocols. Different concentrations of the NO synthase inhibitor Smethylisothiourea sulfate (SMT, Beyotime, Shanghai, China) were used to inhibit the secretion of NO in 
ADSCs-CM and the medium (CM + SMT) was used to culture microglia so as to analyze whether nitric oxide (NO) was one of the main inhibitory factors secreted by ADSCs. By measuring the concentrations of two major inflammatory cytokines (TNF-a, IL-1 3 ), whether NO had a major role in the inhibitory effect of ADSCs on microglia inflammation was analyzed.

\section{Microglia phenotype analysis}

Flow cytometry was used to investigate the levels of proteins CD68 and CD11b on the surface of microglia. After culturing the cells in the four experimental groups for $48 \mathrm{~h}$, the microglia were collected and resuspended in flow cytometry staining buffer (BD Biosciences) for $20 \mathrm{~min}$ at $4^{\circ} \mathrm{C}$. The cells were stained with antibodies against CD68 (FITC-labeled; AbD Serotec, Oxford, UK) and CD11b (FITC-labeled; $\mathrm{BD}, \mathrm{CA}, \mathrm{USA}$ ) for $30 \mathrm{~min}$ at $4^{\circ} \mathrm{C}$ in the dark. Each sample was washed twice with $2 \mathrm{~mL}$ of flow cytometry staining buffer and resuspended in $500 \mu \mathrm{L}$ of staining buffer. Flow cytometry was performed using a BD FACS Canto II (clinical type), and the results were analyzed using BD FACSDiva software (all BD Bioscience). Control experiments were performed using isotype-matched immunoglobulins (BioLegend) to determine the specificity of antibody signals against specific antigens.

\section{Wound healing assay}

The cell migration was evaluated using wound healing assay. Briefly, the microglia were seeded at a density of $5 \times 10^{4}$ cells per well in 6 -well plates and were to $80 \%$ confluence in complete media. After treating with mitomycin $C$ to inhibit proliferation, the monolayer was scratched using a $200-\mu \mathrm{L}$ pipette tip to create a perpendicular wound. Thereafter, the cells were washed with PBS to eliminate cell debris, and the incubation was continued for an additional $24 \mathrm{~h}$ in DMEM or ADSCs-CM containing $1 \% \mathrm{FBS}$, with or without $1 \mu \mathrm{g} / \mathrm{mL}$ LPS. The scratched areas were photographed 0 and $24 \mathrm{~h}$ after wounding using computer-assisted microscopy. The wound closure was calculated by measuring the remaining space in the microscopic images.

\section{Transwell migration assay}

Transwell membrane filters (8- $\mu$ m pore; Corning Costar, MA, USA) were used to assess the role of ADSCs in the migration of microglia. First, $1 \times 10^{4}$ microglia were plated into the upper chamber of the Transwell with serum-free media. This upper chamber was then placed within the bottom well containing $1 \% \mathrm{FBS}$ medium with $2 \times 10^{4} \mathrm{ADSCs}$ (co-culture group) or without ADSCs (control group). Also, the microglia were incubated in ADSCs-CM containing $1 \%$ FBS in the bottom well to see whether the migration was influenced by ADSCs-CM. After incubating at $37^{\circ} \mathrm{C}$ for 12 and $24 \mathrm{~h}$, the nonmigrating cells were carefully swabbed off the upper surface of the membrane. The cells on the lower surface of the membrane were stained using crystal violet (Sigma-Aldrich). The cell migration was measured by counting the number of stained cells on the membrane using a Leica DMIL microscope.

\section{Phagocytosis assay}

The phagocytic capacity of microglia was examined by incubating microglia with fluorescently labeled latex beads. Briefly, $1 \times 10^{5}$ microglia were plated into 24 -well plates containing $500 \mathrm{~mL}$ of DMEM or 
ADSCs-CM with or without $1 \mu \mathrm{g} / \mathrm{mL}$ LPS. Twenty-four hours later, $5 \mathrm{~mL}$ of green fluorescence-labeled latex beads (L3030, Sigma, USA) were added to each well, and the incubation was continued for $4 \mathrm{~h}$. At the end of this stage, the cells were fixed with $4 \%$ paraformaldehyde for 20 min and washed with PBS to remove serum and unbound beads. Finally, the cells were stained with DAPI, and whether microglia were able to phagocytose fluorescent beads was observed under a fluorescence microscope. Phagocytic activity was quantified by the number of beads phagocytosed by microglia using the Image $\mathrm{J}$ Analysis System. Phagocytic index $=$ (total number of fluorescent beads phagocytosed by 100 microglia)/100.

\section{Statistical analysis}

Data were expressed as the mean \pm standard deviation. A comparison between multiple groups was performed using a one-way analysis of variance followed by an unpaired Student's $t$ test. $P$ values $<0.05$ were considered significant. All analyses were performed using the Statistical Package for the Social Sciences, version 17.0 (SPSS, IL, USA).

\section{Results}

Activated microglia promoted neuronal apoptosis, whereas ADSCs inhibited neuronal apoptosis.

TUNEL assays were used in this study to evaluate the effects of activated microglia on neuronal apoptosis (Fig. 1A). As described earlier, the microglia were activated via application of $1 \mu \mathrm{g} / \mathrm{mL}$ LPS before co-culturing with neurons. The results of the TUNEL assay clearly demonstrated that neuronal apoptosis was significantly increased at the indicated time points following incubation with activated microglia (Fig. 1B, LPS group vs control group, $P<0.05$ after 12, 24, and $48 \mathrm{~h}$ ). Treatment with ADSCs-CM significantly inhibited neuronal apoptosis (Fig. 1B, ADSCs-CM group vs, control group, $P<0.05$ after 12, 24 , and $48 \mathrm{~h}$ ). Compared with the LPS group, the ADSCs-CM + LPS group showed reduced neuronal apoptosis (Fig. 1B, P $<0.05$ after 12, 24 and $48 \mathrm{~h}$ ). These results indicated that inflammation caused by microglial cells exerted a harmful effect on neurons, and that ADSCs or ADSCs-CM could exert protective effects on neurons by reducing the rate of apoptosis.

\section{ADSCs inhibited microglia secretion.}

The study further analyzed cytokine and chemokine production by microglia cultured in ADSCs-CM to better characterize the inhibitory effect of ADSCs on microglia inflammation. The supernatants were collected after subjecting microglia to different treatments described earlier. The levels of six cytokines and chemokines (TNF- $a$, IL-1 $\beta$, IL-10, MCP-1, RANTES, and MIP-2) were determined using Bio-Plex Pro assays. The results showed that the microglia produced more TNF-a, IL-1 $\beta$, RANTES, and MIP-2 than those in the control group after incubation in $1 \mu \mathrm{g} / \mathrm{mL}$ LPS $(P<0.001$ for each cytokine), while ADSCs-CM significantly suppressed the secretion of these factors (Fig. 2A-2D). The levels of anti-inflammatory cytokines such as IL-10 and chemokines such as MCP-1 were also significantly higher in LPS-treated microglia compared with the control group (Fig. 2E and 2F, P<0.001). An analysis of the difference between the ADSCs-CM and control groups revealed that the supernatant obtained from ADSCs-CM- 
treated groups contained more IL-10 and MCP-1, indicating that ADSCs promoted the production of large quantities of these factors. In some ADSCs-CM, SMT was added to inhibit the secretion of NO. The ability of microglia to secrete inflammatory cytokines cultured in these supernatants significantly increased (Fig. 3).

\section{ADSCs inhibited the expression of activated microglia receptors.}

Flow cytometry was used to detect the expression of microglia surface receptors CD68 and CD11b (Fig. 4). When microglia in the four groups were treated for $48 \mathrm{~h}$, the expression of the microglia surface receptor CD68 significantly increased in the LPS group compared with the control group $(P<0.05)$. However, the expression of CD68 was significantly lower in the ADSCs-CM group than in the control group $(P<0.05)$. In addition, the cells cultured in the CM + LPS group showed lower expression of CD68 compared with the cells cultured in the LPS group $(P<0.05)$. The study also found that the expression of CD11b on the cell surface was not significantly different among the four groups. CD68 is a typical protein marker of neurotoxic pro-inflammatory M1 microglia. Hence, the results showed that ADSCs therapy was associated with a decrease in the number of brain M1 microglia.

\section{ADSCs significantly decreased distances of microglia migration in wound healing assay.}

Microglia are known to be highly migratory cells as other macrophage populations; they can quickly move to the sites of brain damage after detecting small homeostatic disturbances. Microglia migration during wound healing in vivo was mimicked using a wound healing assay, and whether ADSCs could affect this important property of microglia was investigated. As shown in Fig. 5, the control microglia and LPS-treated microglia spontaneously migrated and filled $36.18 \pm 4.75 \%$ and $59.87 \pm 10.44 \%$ of the wounded region, respectively, after $24 \mathrm{~h}$ of culture. In contrast, the migration ability of microglia cultured with ADSCs-CM was obviously weakened. The distance of cell migration in the ADSCs-CM groups (ADSCs-CM and CM + LPS groups) significantly decreased $24 \mathrm{~h}$ after scratching, and the wound closure was reduced by $65.21 \%$ and $64.10 \%$ compared with control and LPS-treated microglia, respectively. It was speculated that ADSCs might secrete some extracellular effector factors with the capacity to suppress microglial migration.

\section{ADSCs reduced the average number of migrating microglia in the Transwell assay.}

Transwell migration assays were performed to further confirm the inhibitory effect of ADSCs on microglial migration. First, the microglia were seeded into the upper chambers and then placed into bottom chambers containing either ADSCs (co-culture group) or no cells (control group). The number of microglia that migrated from the upper surface of the membrane to the lower surface was tested. The bottom wells were also filled with ADSCs-CM instead of ADSCs (ADSCs-CM group) to assess whether the number of migratory microglia could be influenced by molecules secreted by ADSCs into the extracellular media alone. As shown in Fig. 6, fewer cells in the co-culture group passed through the membrane compared with cells in the control group (co-culture group vs control group, $P<0.01$ after 12 and $24 \mathrm{~h}$ ). A sharp decline in chemotactic migration toward the serum was found in cells in the ADSCs-CM group 
compared with the cells in the control group (ADSCs-CM group vs control group, $P<0.05$ after $12 \mathrm{~h}$ and $P$ $<0.05$ after $24 \mathrm{~h})$.

\section{ADSCs inhibited microglial phagocytosis.}

The phagocytosis of latex beads by microglia was observed under a fluorescence microscope. As shown in Fig. 7, microglia activated with $1 \mu \mathrm{g} / \mathrm{mL}$ LPS (LPS group) phagocytosed more green fluorescent latex beads compared with the microglia in the control group. Meanwhile, the microglia cultured in ADSCs-CM phagocytosed fewer latex beads compared with the microglia in the control group. The microglia cultured in ADSCs-CM with LPS (CM + LPS group) showed depressed phagocytic ability compared with the microglia cultured in LPS alone (LPS group).

\section{Discussion}

This study investigated the anti-inflammatory and immunomodulatory properties of ADSCs by culturing microglia with ADSCs or ADSCs-CM. Following co-culture with ADSCs or ADSCs-CM for different time periods, the microglia showed decreased secretion of inflammatory cytokines, reduced migration, weakened phagocytic capacity, and decreased the expression of CD68. Furthermore, primary hippocampal neurons were cultured with activated microglia in a Transwell system co-cultured with ADSCs or ADSCs-CM to examine the protective effect of ADSCs on the neurons. ADSCs could significantly inhibit the apoptosis of neurons in the inflammatory state.

The functional effects of microglia in the normal and damaged nervous system have been explored because of their key role in the brain. In the normal brain, the microglia exist in a resting state; upon a perception of a microbial attack or tissue lesion, they immediately initiate an inflammatory reaction to keep tissues in a stable condition and start repair processes [29]. Hence, microglia activation is an essential step in an efficient CNS immune response. Nevertheless, the inflammatory response caused by microglia may impair nerve cells as a result of overactivated inflammatory reactions that are not well controlled. A previous study showed that the blockade of microglial activation using anti-inflammatory drugs such as minocycline could significantly alleviate the pathological process of many neurodegenerative diseases [30]. Thus, attenuating detrimental microglial activity could demonstrate therapeutic efficacy against neuronal lesions and degeneration. ADSCs are a multipotent cell type efficient in immune reaction regulation through cell-cell interactions and their secreted molecules. Many studies confirmed the therapeutic impacts of ADSCs and conditioned media on different inflammatory diseases. ADSCs have been demonstrated to reduce the expression of pro-inflammatory cytokine genes in experimental acute lung injury, myocardial infarction, and acute renal failure. However, reports on whether ADSCs can treat inflammatory diseases of the CNS by inhibiting microglial activity are recently emerging [31].

In the present study, data from Bio-Plex Pro tests demonstrated that ADSCs significantly inhibited the expression of cytokines, such as TNF- $\alpha$, IL-1 $\beta$, RANTES, and MIP-2, induced by microglia. TNF- $\alpha$ and IL-1 $\beta$ are neuroinflammatory factors that facilitate the degeneration of dopamine neurons [32]. Thus, a 
reduction in the number of TNF- $\alpha$ and IL-1 $\beta$ might present a potential therapeutic method for treating different neurodegenerative diseases. The results of our study demonstrated that ADSCs could inhibit neuronal apoptosis, and the inhibition was probably achieved by inhibiting the level of neuroinflammatory factors. Other scholars have also proved that up-regulating TSG-6 expression of ADSCs could inhibit the release of inflammatory factors mediated by BV2 microglia, which was similar to our experimental results [31]. The NO inhibitor SMT was used to inhibit the secretion of NO by ADSCs so as to determine whether NO was one of the main inhibitory factors secreted by ADSCs. The present study showed that the ability of microglia to secrete TNF-a and IL-1 $\beta$ was significantly enhanced when NO was inhibited in conditioned medium of cultured microglia. Other studies also reported that NO at high concentrations could suppress the phosphorylation of signal transducer and activator of transcription 5 in T cells and promote immune cell apoptosis in vitro, which was similar to present findings [33]. The levels of chemokine RANTES, which had the capacity to activate T lymphocytes, increased significantly after brain damage. T lymphocytes have been shown to infiltrate the brain following ischemic stroke, releasing proinflammatory cytokines and cytotoxic substances, which contribute to early inflammation and brain damage [34]. The reduction of T-lymphocyte infiltration in the initial stages following injury is important for improving the prognosis of ischemic stroke. MIP-2 has been shown to contribute to neutrophil infiltration and subsequent secondary neurodegeneration following TBl; an experimental model of TBI demonstrated that the levels of MIP-2 increased rapidly after injury [35]. The present study found that the secretion of RANTES and MIP-2 by activated microglia significantly increased. The ability of ADSCs to suppress the formation of RANTES and MIP-2 indicated that ADSCs might also have a negative effect on diseases mediated by activated microglia. The present study also demonstrated that the supernatants derived from ADSCs contained a large amount of MCP-1 and IL-10. MCP-1 is the most effective chemoattractant for monocytes, macrophages, and microglia, which play significant roles in mediating secondary brain damage following TBI. IL-10 is increasingly regarded as an effective antiinflammatory and immunosuppressive cytokine that regulates the proliferation and differentiation of various immune cell types [36, 37]. Studies confirmed that the number of M2-type microglia increased when microglia were cultured with IL-10. These type of cells were neuroprotective, as they were involved in phagocytosis and damage repair [38,39]. Therefore, it was believed that the large amount of IL-10 secreted by ADSCs might be one of the main mechanisms underlying the inhibition of the activity of microglia. The results of the present study demonstrated the inhibitory effect of ADSCs-CM on microglial inflammation. This inhibitory effect was thought to be achieved mainly through the secretion of inhibitory substances by ADSCs, which was similar to a previous study by Feng et al., which proved that exosomes from ADSCs alleviated neural injury caused by microglial activation [40].

Apart from the production of neurotoxic mediators, faulty phagocytosis of normal neurons by microglia is another cause of neurodegenerative disorders [6]. Under normal circumstances, necrotic or apoptotic neurons are rapidly eliminated via the phagocytosis of microglia. Nevertheless, in inflammatory circumstances, microglia show a marked increase in phagocytosis, when they indiscriminately ingest dead and normal neurons. The present study provided evidence that ADSCs significantly inhibited the phagocytosis of activated microglia, indicating that ADSCs could inhibit neuronal cell death induced by 
microglia to some extent. The movement of microglia to the lesions is considered a key event in brain inflammation [41]. Mounting evidence demonstrates that microglia migrate rapidly toward the injury site in response to focal cerebral ischemic insults. Sustained migration and accumulation of microglial cells have a detrimental effect, contributing to neurodegeneration, in which neuronal loss is accompanied by increased neuroinflammatory conditions. Hence, stem cell-based therapeutic treatments that reduce microglial migration can further prevent inflammation and the death of related neurons. Such therapies have attracted increasing attention, and many studies have been carried out to evaluate their efficacy. In this study, both the wound healing assay and the Transwell migration test demonstrated that ADSCs could significantly reduce the migration of microglia, suggesting that ADSCs could reduce neuronal damage caused by microglia migrating to the lesion site. The present study also proved that ADSCs showed an inhibitory effect on the surface expression of CD68 receptors. As CD68 is a typical protein marker of neurotoxic pro-inflammatory M1 microglia, the results showed that ADSCs therapy was associated with a decrease in the brain M1 microglia ratio, suggesting that ADSCs could significantly inhibit neuroinflammation caused by microglia.

Finally, the study found that the number of apoptotic neurons increased after culturing neurons with activated microglia for different time periods. However, neuronal apoptosis significantly reduced when neurons were cultured in ADSCs-CM, compared with incubation with control media. This result suggested that activated microglia had a deleterious effect on neurons, but this effect could be inhibited by treatment with ADSCs-CM. As maintaining neuronal survival after injury is critical for CNS function, it was believed that ADSCs-mediated neuronal protection might be a promising therapeutic strategy.

The present study demonstrated that ADSCs inhibited not only the ability of microglia to secrete inflammatory factors but also the phagocytosis and migration of microglia. Treatment with ADSCs also reduced neuronal apoptosis under inflammatory conditions. The study focused on the inhibitory effect of ADSCs on the inflammatory level of microglia, which was different from previous studies showing that ADSCs exerted a therapeutic effect by differentiating into neurons or producing trophic factors. ADSCs had the ability to suppress microglial inflammation in the CNS, demonstrating that ADSCs could be used as effective tools to control diseases associated with microglial inflammation.

\section{Declarations}

\section{Funding statement}

This work was supported by grants from the Natural Science Fund of Yangzhou City (No. YZ2017114 and YZ2017121), and the Social Development Funds of Yangzhou City (No. YZ2017070 and YZ2018088).

\section{Compliance with Ethical Standards}

\section{Conflict of Interest}

The authors declare that they have no conflict of interest. 


\section{Consent to participate}

Not applicable.

\section{Consent for Publication}

Not applicable.

\section{Availability of data and material}

All data are fully available without restriction.

\section{Authors' contributions}

Conceived and designed the experiments: Ke Yan, Yong Zhen, Jiarong Bian. Performed the experiments: Ke Yan, Yong Zhen, Liang He, Jiarong Bian, Bingwei Song, Jiawei He, Linhai Shen. Analyzed the data: Yong Zhen, Jiarong Bian, Xinzhi Zhou, Lun Dong. Wrote the paper: Ke Yan, Jiarong Bian. Paper revision: Yong Zhen. All authors read and approved the final manuscript.

\section{References}

1. Rothhammer V, Quintana FJ (2015) Role of astrocytes and microglia in central nervous system inflammation. Seminars in Immunopathology 37:575-576.

2. Walter L, Neumann H (2009) Role of microglia in neuronal degeneration and regeneration. Seminars in Immunopathology 31:513-525.

3. Ghoshal A, Das S, Ghosh S, Mishra MK, Sharma V, Koli P, Sen E, Basu A (2007) Proinflammatory mediators released by activated microglia induces neuronal death in Japanese encephalitis. Glia 55:483-496.

4. Block ML, Zecca L, Hong JS (2007) Microglia-mediated neurotoxicity: uncovering the molecular mechanisms. Nature Reviews Neuroscience 8:57-69.

5. Wang GQ, Li DD, Huang C, Lu DS, Zhang C, Zhou SY, Liu J, Zhang F (2018) Icariin Reduces Dopaminergic Neuronal Loss and Microglia-Mediated Inflammation in Vivo and in Vitro. Frontiers in Molecular Neuroscience 10:3389.

6. Neher JJ, Neniskyte U, Zhao JW, Bal-Price A, Tolkovsky AM, Brown GC (2011) Inhibition of microglial phagocytosis is sufficient to prevent inflammatory neuronal death. Journal of Immunology 186:4973-4983.

7. Walker DG, Whetzel AM, Lue LF (2014) Expression of suppressor of cytokine signaling genes in human elderly and Alzheimer's disease brains and human microglia. Neuroscience 302:121-137.

8. Lynch MA (2009) The multifaceted profile of activated microglia. Molecular Neurobiology 40:139156. 
9. Yrjanheikki J, Keinanen R, Pellikka M, Hokfelt T, Koistinaho J (1998) Tetracyclines inhibit microglial activation and are neuroprotective in global brain ischemia. Proceedings of the National Academy of Science of the United States of America 95:15769-15774.

10. Frank-Cannon TC, Alto LT, McAlpine FE, Tansey MG (2009) Does neuroinflammation fan the flame in neurodegenerative diseases? Molecular Neurodegeneration 4:47-59.

11. Bodo C (2010) Neurodegenerative diseases: Microglia's little helper. Nature Reviews Neuroscience 11:297.

12. Zhou Y, Wang Y, Monika K, Jin JH (2005) Microglial Activation Induced by Neurodegeneration. Molecular and Cellular Proteomics 4:9.

13. Shi Y, Su J, Roberts A, Shou P, Rabson A, Ren G (2012) How mesenchymal stem cells interact with tissue immune responses. Trends in Immunology 33:136-143.

14. Uccelli A, Moretta L, Pistoia V (2008) Mesenchymal stem cells in health and disease. Nature Reviews Immunology 8:726-736.

15. Giuseppina M, Carella S, Ceccarelli S, Marchese C, Scuderi N (2016) The Use of Human AdiposeDerived Stem Cells in the Treatment of Physiological and Pathological Vulvar Dystrophies. Stem Cells International 2016:2561416.

16. Wang L, Deng J, Li G, Wang J, Xiang B, Kashour T, Arora R, Gruwel M, Glogowski M, Tomanek B, Deslauriers R, Tian G (2007) Adipose-derived stem cells significantly improve cardiac function of the infarct rat hearts. Journal of Molecular and Cellular Cardiology 42:s97.

17. Jung PY, Ryu H, Rhee KJ, Hwang S, Lee CG, Gwon SY, Kim J, Kim J, Yoo BS, Baik SK, Bae KS, Eom YW (2018) Adipose tissue-derived mesenchymal stem cells cultured at high density express IFN-b and suppress the growth of MCF-7 human breast cancer cells. Cancer letters 440:202-210.

18. Jha KA, Pentecost M, Lenin R, Klaic L, Elshaer SL, Gentry J, Russell JM, Reiner A, Beland A, Jotterand V, Sohl N, Gangaraju R (2018) Concentrated Conditioned Media from Adipose Tissue Derived Mesenchymal Stem Cells Mitigates Visual Deficits and Retinal Inflammation Following Mild Traumatic Brain Injury. International Journal of Molecular Sciences 19:2016.

19. Ferrero-Gutierrez A (2011) Transplantation of adipose derived stem cells (ADSCs) and olfactory ensheathing cells (OECs) cultured in a new scaffold for the repair of spinal cord injury (SCI) in rats: 6 . British Journal of Surgery 98:s2.

20. Safford KM, Hicok KC, Safford SD, Wilkison WO, Halvorsen YD, Gimble JM, Rice HE (2002) Neurogenic differentiation of murine and human adipose-derived stromal cells. Biochemical and Biophysical Research Communications 294:371-379.

21. Jeong SR, Kwon MJ, Lee HG, Joe EH, Lee JH, Kim SS, Suh-Kim H, Kim BG (2012) Hepatocyte growth factor reduces astrocytic scar formation and promotes axonal growth beyond glial scars after spinal cord injury. Experimental Neurology 233:312-322.

22. Wilkins A, Kemp K, Ginty M, Hares K, Mallam E, Scolding N (2009) Human bone marrow-derived mesenchymal stem cells secrete brain-derived neurotrophic factor which promotes neuronal survival in vitro. Stem Cell Research 3:63-70. 
23. Koh SH, Baik W, Noh MY, Cho GW, Kim HY, Kim KS, Kim SH (2012) The functional deficiency of bone marrow mesenchymal stromal cells in ALS patients is proportional to disease progression rate. Experimental Neurology 233:472-480.

24. Sasaki M, Radtke C, Tan AM, Zhao P, Hamada H, Houkin K, Honmou O, Kocsis JD (2009) BDNFhypersecreting human mesenchymal stem cells promote functional recovery, axonal sprouting, and protection of corticospinal neurons after spinal cord injury. Journal of Neuroscience 29:1493214941.

25. Chan TM, Chen JYR, Ho LI , Lin HP, Hsueh KW, Liu DD, Chen YH, Hsieh AC, Tsai NM, Hueng DY (2014) ADSC Therapy in Neurodegenerative Disorders. Cell transplantation 23:549-557.

26. Azim H, Iraj R, Parichehr P (2013) Promotion of Remyelination by Adipose Mesenchymal Stem Cell Transplantation in A Cuprizone Model of Multiple Sclerosis. Cell Journal (Yakhteh) 15:142-151.

27. Ma T, Gong K, Ao Q, Yan YF, Song B, Huang HY, Zhang XF, Gong YD (2013) Intracerebral Transplantation of Adipose-Derived Mesenchymal Stem Cells Alternatively Activates Microglia and Ameliorates Neuropathological Deficits in Alzheimer's Disease Mice. Cell Transplantation 22:113126.

28. Giulian D, Baker TJ (1986) Characterization of ameboid microglia isolated from developing mammalian brain. J Neurosci 6:2163-2178.

29. Jeong HK, Ji K, Min K, Joe EH (2013) Brain Inflammation and Microglia: Facts and Misconceptions. Experimental Neurobiology 22:59-67.

30. Wu DC, Jackson-Lewis V, Vila M, Tieu K, Teismann P, Vadseth C, Choi D, Ischiropoulos H, Przedborski S (2002) Blockade of microglial activation is neuroprotective in the 1-methyl-4-phenyl-1,2,3,6tetrahydropyridine mouse model of Parkinson disease. Journal of Neuroscience 22:1763-1771.

31. Hu Y, Li GG, Zhang Y, Liu N, Zhang P, Pan C, Nie H, Li Q, Tang ZP (2018) Upregulated TSG-6 Expression in ADSCs Inhibits the BV2 Microglia-Mediated Inflammatory Response. BioMed Research International 2018:7239181.

32. Choi Y, Lee MK, Lim SY, Sung SH, Kim YC (2009) Inhibition of inducible NO synthase, cyclooxygenase- 2 and interleukin- $1 \beta$ by torilin is mediated by mitogen-activated protein kinases in microglial BV2 cells. British Journal of Pharmacology 156:933-940.

33. Sato K, Ozaki K, Oh I, Meguro A, Hatanaka K, Nagai T, Muroi K, Ozawa K (2007) Nitric oxide plays a critical role in suppression of T-cell proliferation by mesenchymal stem cells. Blood 109:228-234.

34. Brait VH, Arumugam TV, Drummond GR, Sobey CG (2012) Importance of T lymphocytes in brain injury, immunodeficiency, and recovery after cerebral ischemia. Journal of Cerebral Blood Flow and Metabolism 32:598-611.

35. Rhodes JK, Sharkey J, Andrews PJ (2009) The temporal expression, cellular localization, and inhibition of the chemokines MIP-2 and MCP-1 after traumatic brain injury in the rat. Journal of Neurotrauma 26:507-525.

36. Nauta AJ, Fibbe WE (2007) Immunomodulatory properties of mesenchymal stromal cells. Blood 110:3499-3506. 
37. Pestka S, Krause CD, Sarkar D, Walter MR, Shi YF, Fisher PB (2004) Interleukin-10 and related cytokines and receptors. Annual Review of Immunology 22:929-979.

38. Hjorth E, Zhu M, Toro VC, Vedin I, Palmblad J, Cederholm T, Freund-Levi Y, Faxen-Irving G, Wahlund LO, Basun H, Eriksdotter M, Schultzberg M (2013) Omega-3 fatty acids enhance phagocytosis of Alzheimer's disease-related amyloid-beta42 by human microglia and decrease inflammatory markers. JOURNAL OF ALZHEIMERS DISEASE 35:697-713.

39. Mandrekar-Colucci S, Karlo JC, Landreth GE (2012) Mechanisms underlying the Rapid Peroxisome proliferator-activated receptor- $y$-mediated Amyloid clearance and reversal of cognitive deficits in a murine model of Alzheimer's disease. Journal of Neuroscience 32:10117-10128.

40. Feng NH, Jia YJ, Huang XX (2019) Exosomes from adipose-derived stem cells alleviate neural injury caused by microglia activation via suppressing NF-kB and MAPK pathway. Journal of Neuroimmunology 334:576996.

41. Han Q, Liu S, Li Z, Hu F, Zhang Q, Zhou M, Chen J, Lei T, Zhang H (2013) DCPIB, a potent volumeregulated anion channel antagonist, Attenuates microglia-mediated inflammatory response and neuronal injury following focal cerebral ischemia. Brain research 1542:176-185.

\section{Figures}

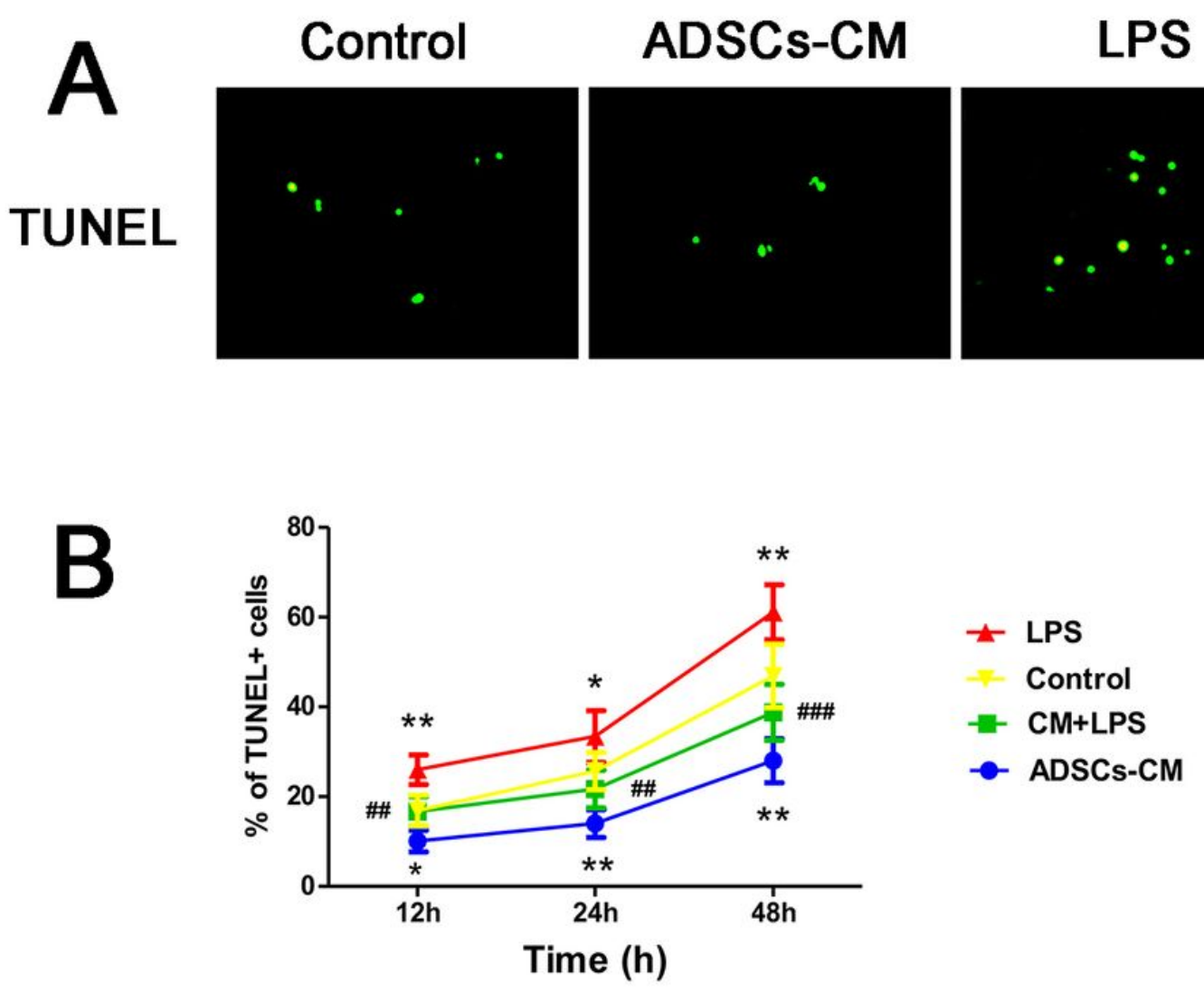

Figure 1 
Effect of ADSCs on neuronal apoptosis. (A) Neuronal apoptosis was observed under a fluorescent microscope (400x magnification). Fewer apoptotic neurons were observed in the ADSCs-CM group compared with the control group, while apoptosis increased in neurons in the LPS group (LPS groups vs. control groups, ${ }^{*} \mathrm{P}<0.01$ at 12,48 hours, ${ }^{*} \mathrm{P}<0.05$ at 24 hours; ADSCs-CM groups vs. control groups, ${ }^{*} P<0.01$ at 24,48 hours, ${ }^{*} P<0.05$ at 12 hours). The number of apoptotic cells significantly reduced in the ADSCs-CM + LPS group compared with the LPS group (CM+LPS groups vs. LPS groups, \#\#P<0.01 at 12, 24 hours, $\# \# \# P<0.001$ at 48 hours). (B) The percentage of apoptotic neurons as a proportion of the total number of neurons in each group. Bars represent the means plus or minus the SD obtained from 10 random fields.

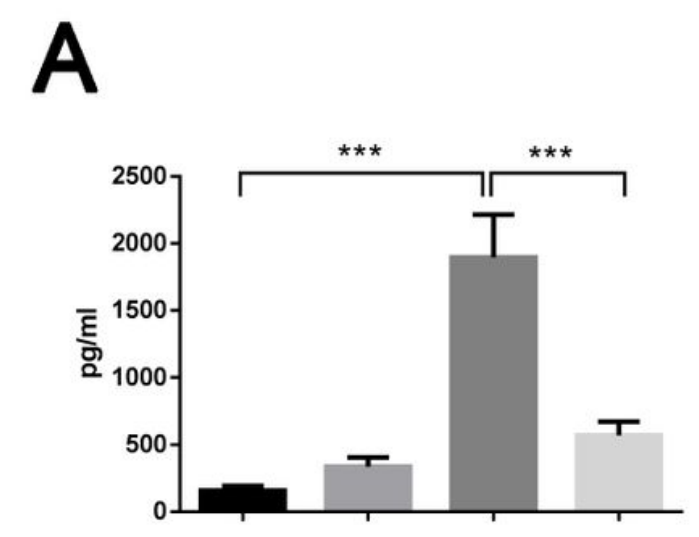

TNF-a

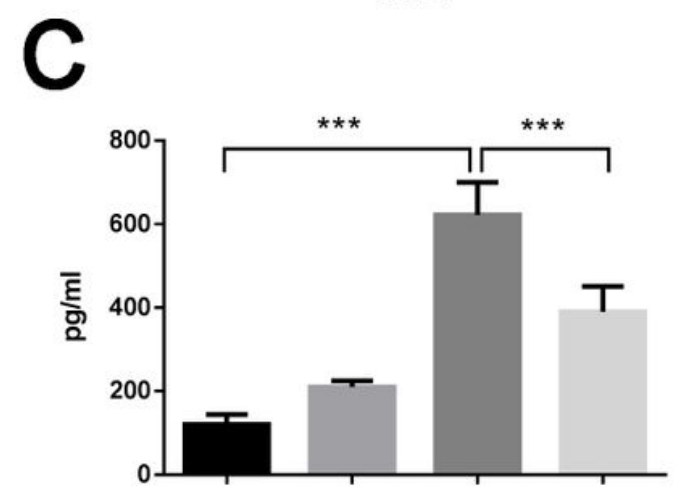

E

RANTES

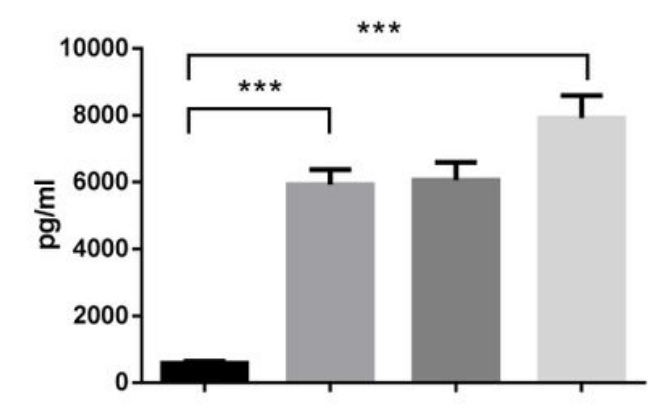

MCP-1

\section{B}

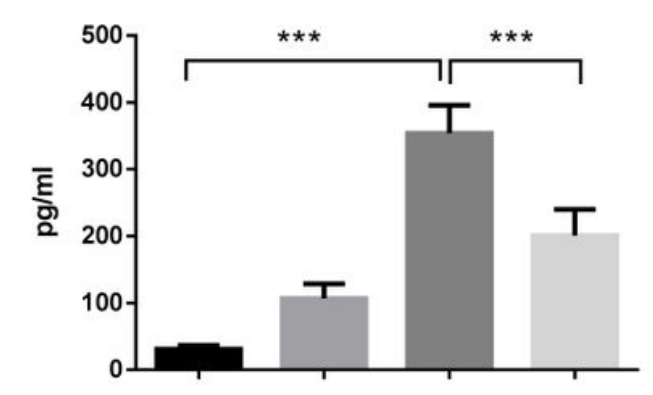

IL-1 $\beta$

D

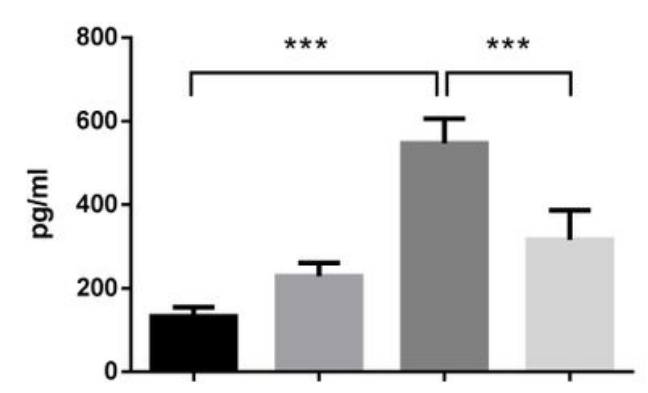

E MIP-2

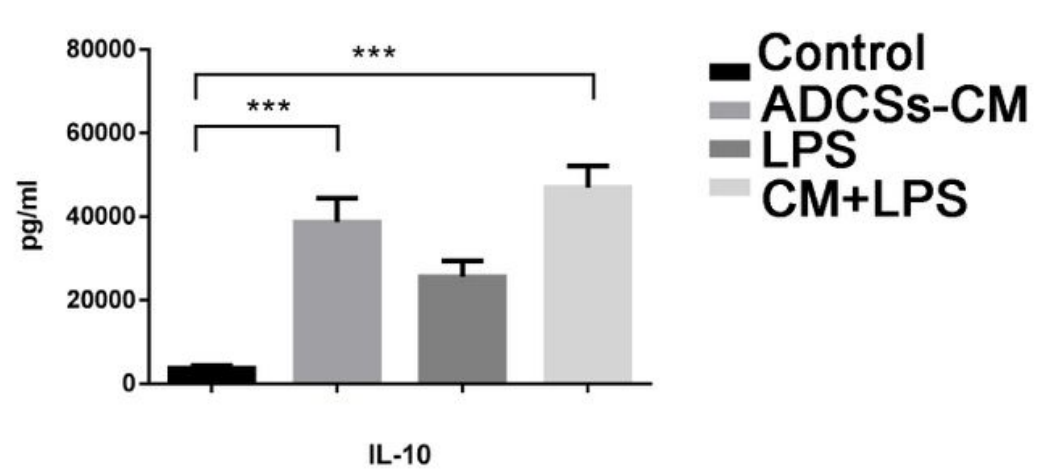

Figure 2 
Cytokine and chemokine production by microglia was analyzed using Bio-Plex Pro assays. (A-D) LPStreated microglia produced more TNF-a, IL-1 $\beta$, RANTES, and MIP-2 compared with control microglia, while treatment with ADSCs-CM significantly inhibited the production of these factors. (E and F) Microglia in the LPS-treated groups produced more MCP-1 and IL-10 compared with control microglia. The supernatant derived from the ADSCs-CM-treated group also contained more MCP-1 and IL-10 compared with that from the control group. Bars represent means plus or minus SD obtained from six independent experiments. A P value of less than $0.05\left(^{*}\right)$, less than $0.01\left(^{(\star *}\right)$, and less than $0.001\left(^{\star \star \star}\right)$ was considered statistically significant.

TNF - $a$

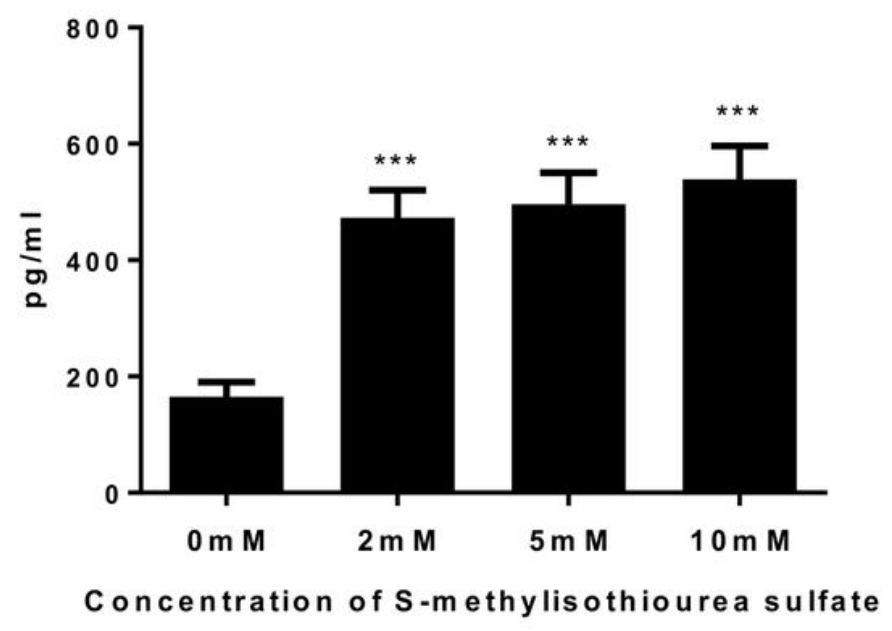

IL $-1 \beta$

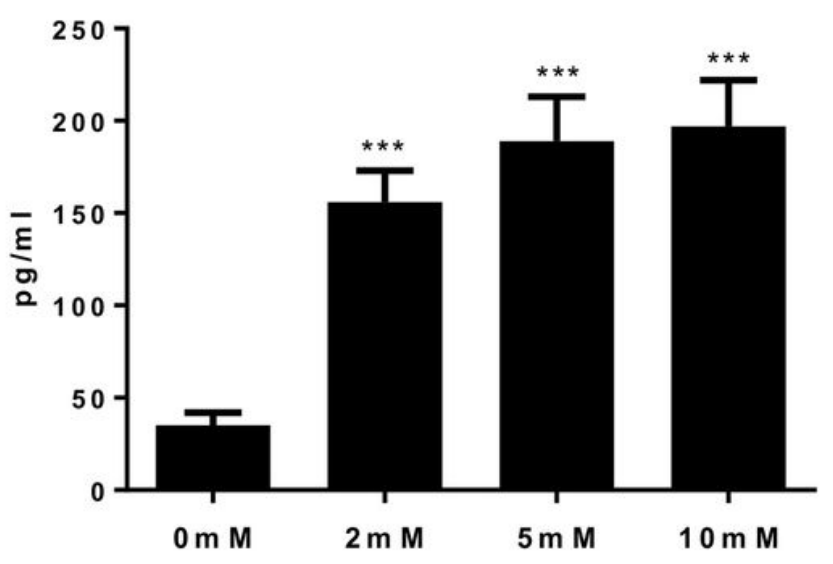

Concentration of S-methylisothiourea sulfate

\section{Figure 3}

Nitric oxide was involved in the inhibition of microglia by ADSCs. The nitric oxide synthase inhibitor Smethylisothiourea sulfate was used to inhibit the secretion of NO in an ADSCs-conditioned medium. The ability of microglia cultured in this medium to secrete TNF- $\alpha$ and IL-1 $\beta$ increased significantly with an increasing concentration of SMT. Bars represent means plus or minus SD obtained from six independent experiments. ${ }^{\star \star \star} \mathrm{P}<0.001$ versus cells cultured in ADSCs-CM plus $0 \mathrm{mM}$ SMT. 
Control
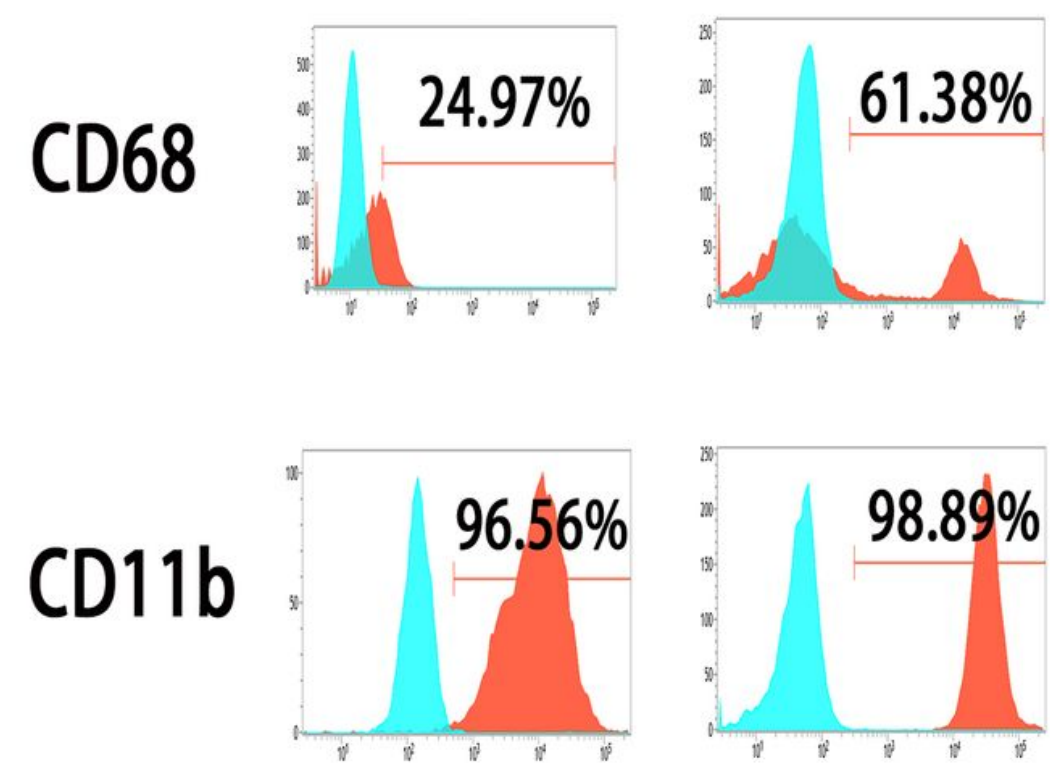

LPS
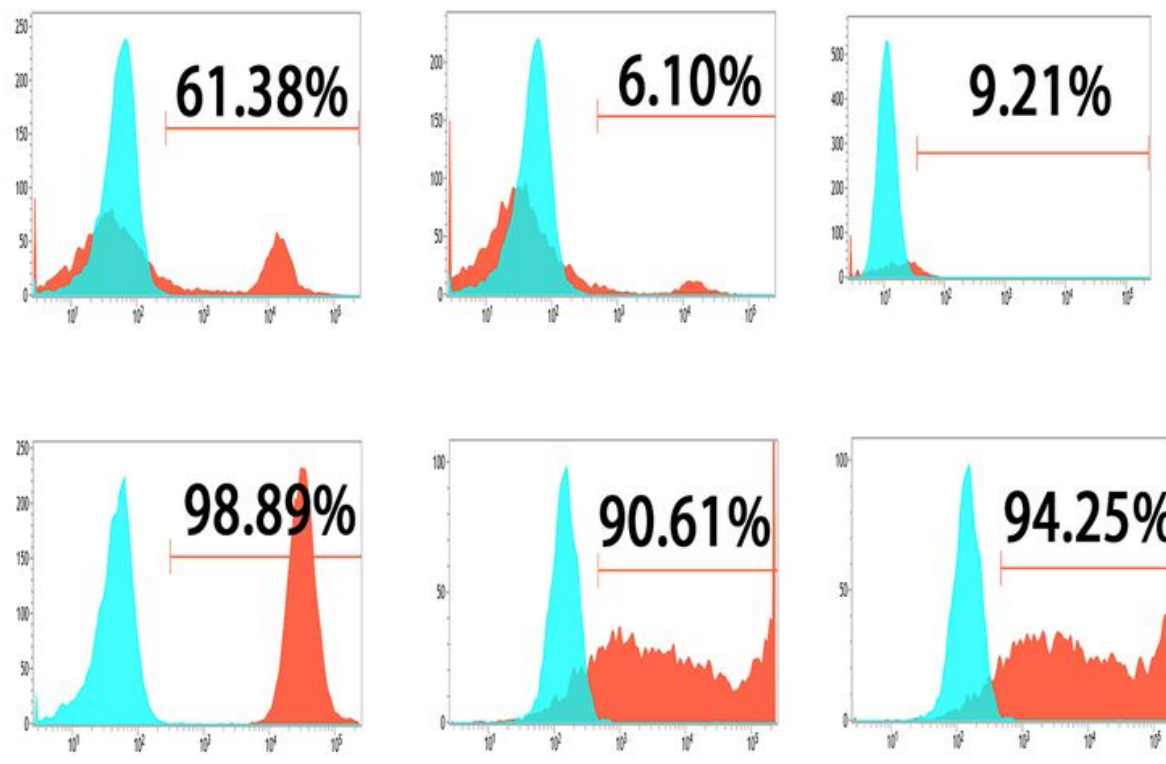

$\mathrm{CM}$

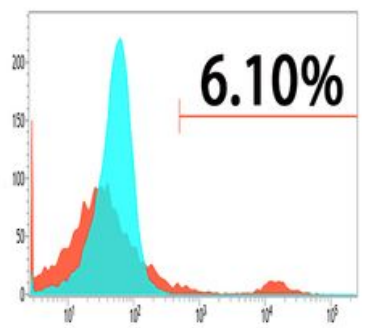

CM+LPS

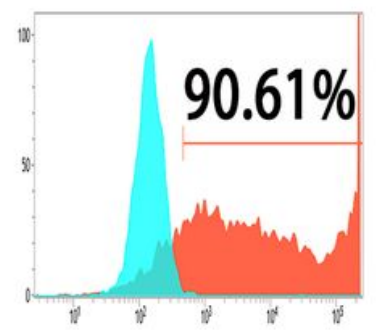

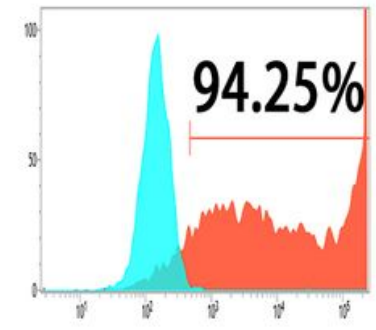

Figure 4

Expression of CD68 and CD11b detected by flow cytometry. Microglia were treated with DMEM or ADSCs$\mathrm{CM}$ with or without $1 \mu \mathrm{g} / \mathrm{mL}$ LPS for $48 \mathrm{~h}$. The expression of the microglial cell surface receptors CD68 and $C D 11 b$ was analyzed by flow cytometry, and the cell surface receptors of each group were tested six times. Profiles represent the expression of activating receptors. Blue, negative controls; numbers, percentages of positive cells. 
Control

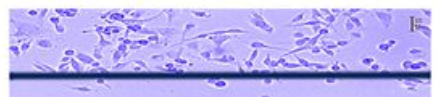

$\mathrm{Oh}$

$24 \mathrm{~h}$
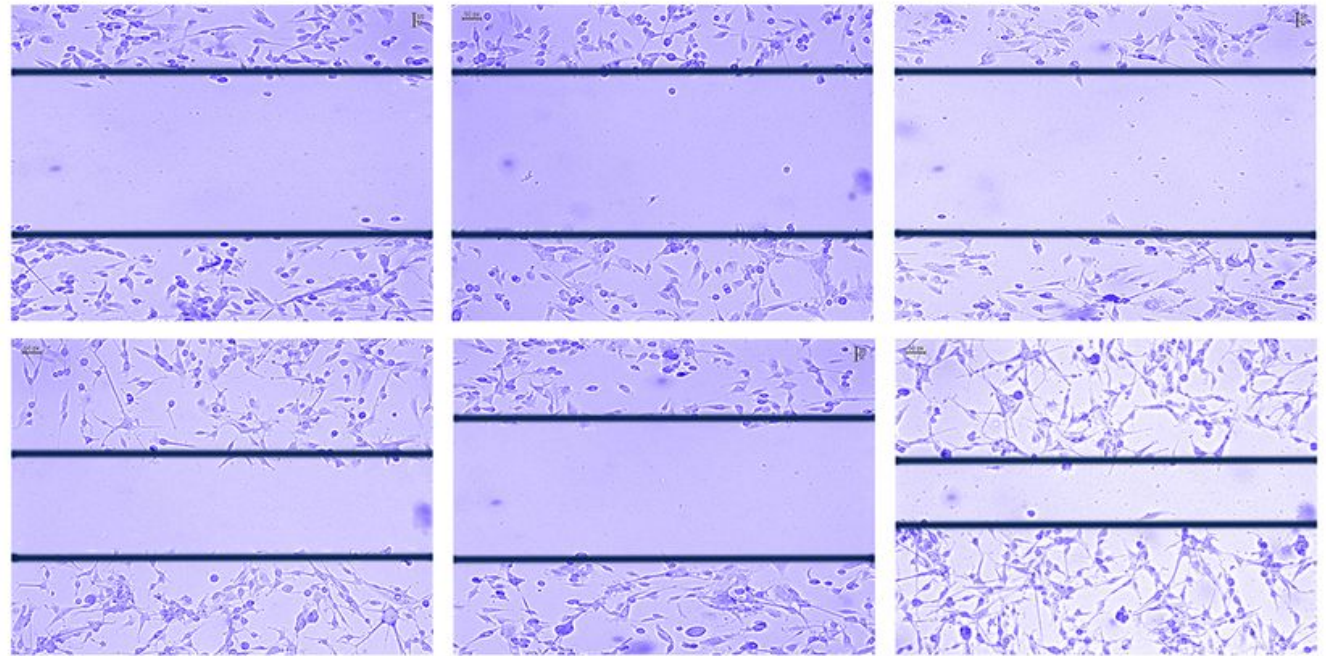

CM+LPS

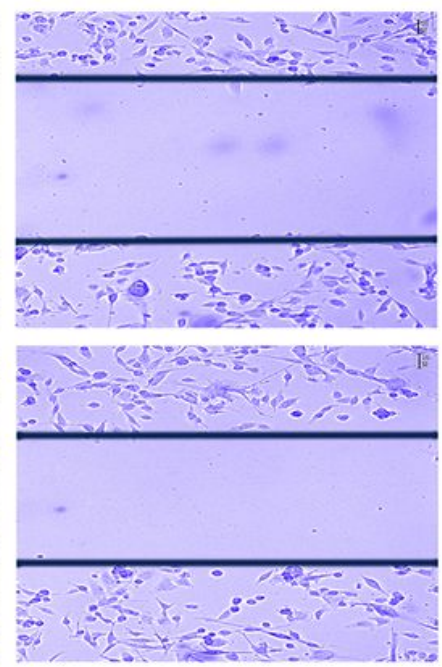

B

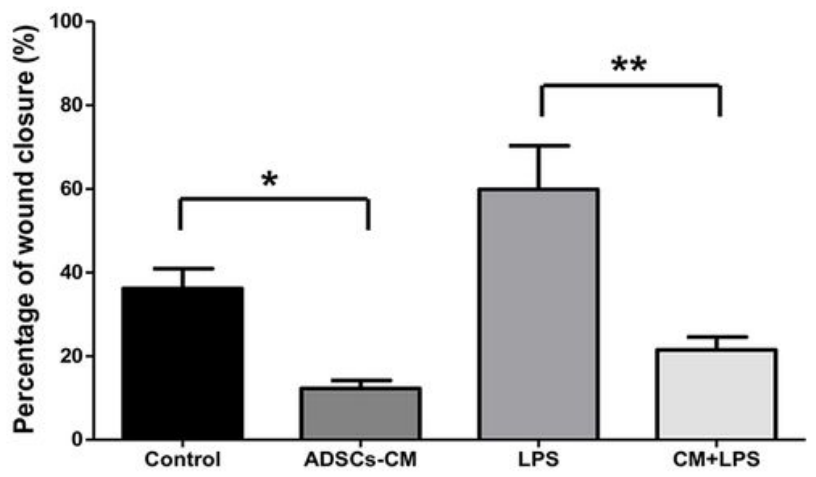

Figure 5

Wound healing assay-based analysis of the effect of ADSCs on microglial migration. (A) Representative images of the wound healing assay showing the effects of ADSCs on the migration of microglia toward the wounded region (200x magnification). The distance between the two black parallel lines after $0 \mathrm{~h}$ is the initial wound size/distance. The distance between the black parallel lines (wound size) after $24 \mathrm{~h}$ of cell migration is also shown. (B) Quantification of wound closure. The percentage of wound closure by microglia after $24 \mathrm{~h}$ in the four groups is shown. Note: CM-treated microglia showed significantly decreased wound closure compared with control microglia and LPS-treated microglia. Bars represent means plus or minus SD obtained from six independent experiments. ${ }^{\star} \mathrm{P}<0.05,{ }^{\star *} \mathrm{P}<0.01$. 
Control

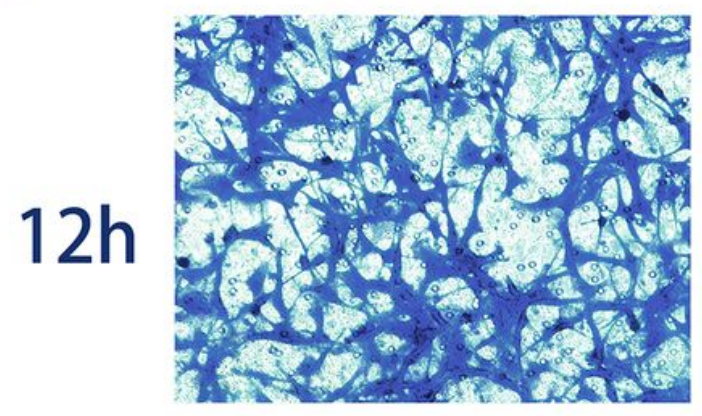

$24 \mathrm{~h}$

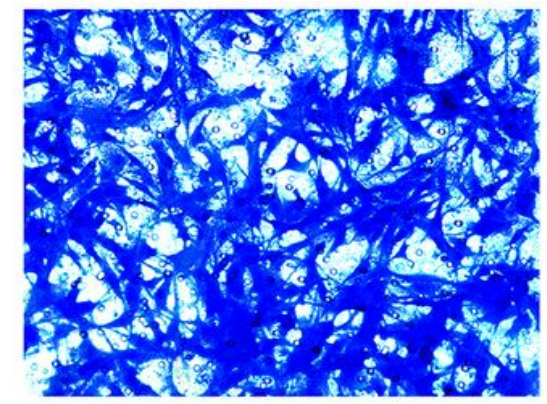

ADSCs-CM
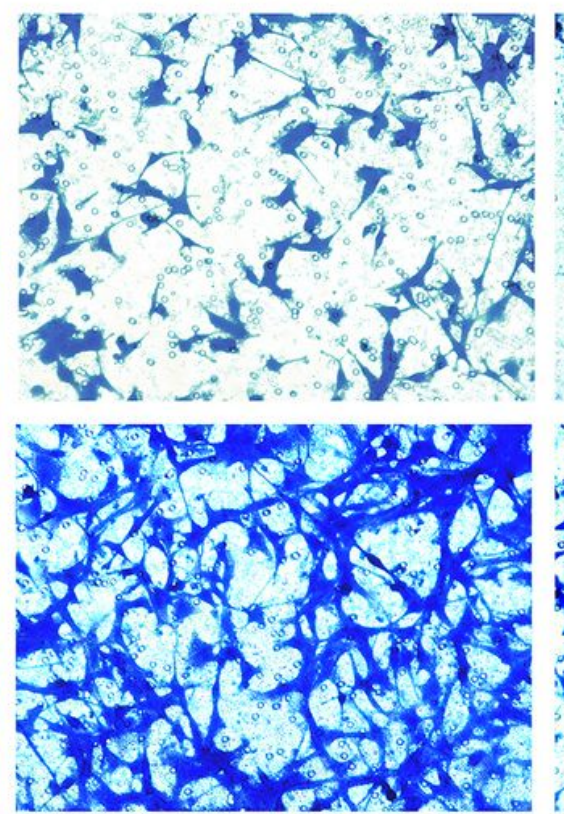

Coculture
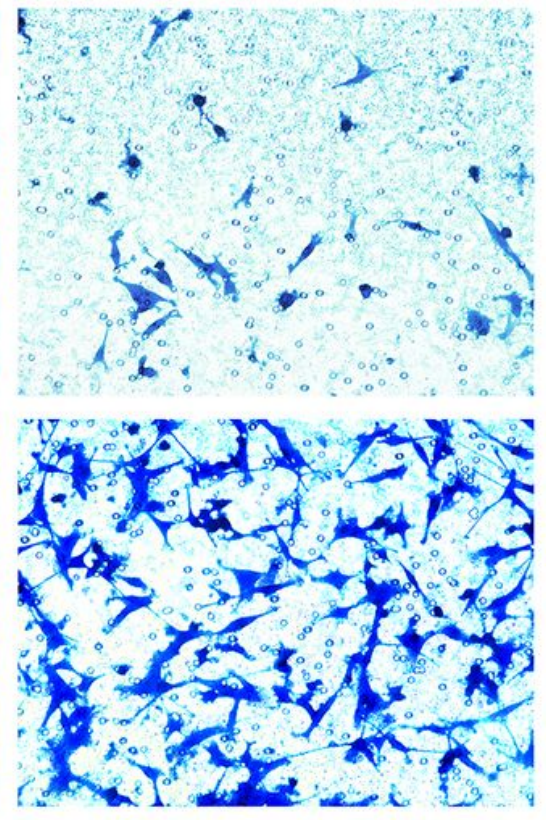

B

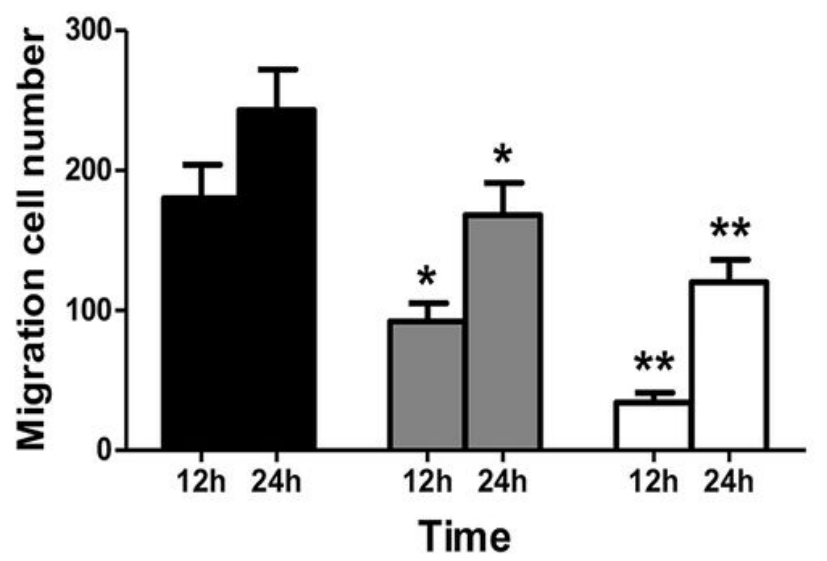

Control

ADSCs-CM

$\square$ Co-culture

Figure 6

Transwell migration assay-based analysis of the effect of ADSCs on microglial migration. (A) Crystal violet-stained microglia migrated into the lower surface of the polycarbonate membrane inserts $(8-\mu \mathrm{m}$ pore size) 12 and $24 \mathrm{~h}$ after seeding (200x magnification). (B) The average number of migrating microglia per visual field in six random fields (200x magnification; mean \pm SD). Microglia were quantified in the control, co-culture, and ADSCs-CM groups. ${ }^{*} \mathrm{P}<0.05,{ }^{*} \mathrm{P}<0.01$ versus the control group. 

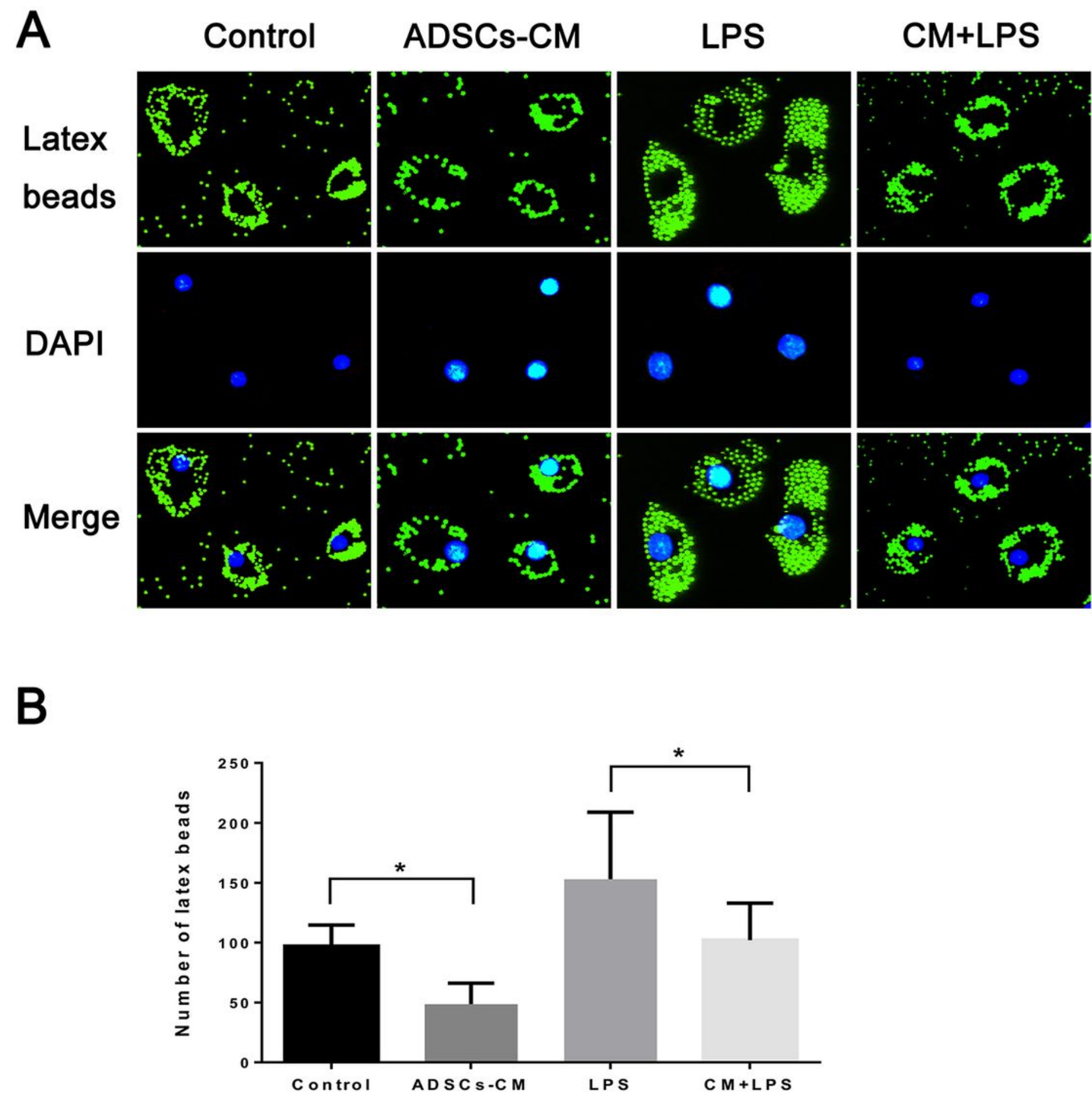

Figure 7

ADSCs inhibited microglial phagocytosis. (A) The capacity of microglia to phagocytose fluorescently labeled latex beads was tested by observing them under a fluorescence microscope (400x magnification). Nuclei were counterstained with DAPI. (B) The bar graph shows that the microglia ingested significantly more latex beads following $4 \mathrm{~h}$ of incubation with LPS ( $1 \mathrm{mg} / \mathrm{mL})$, compared with the untreated control group (LPS group vs control group, ${ }^{\star} \mathrm{P}<0.05$ ), while the phagocytic activity of 
microglia in the CM-treated group was significantly inhibited (ADSCs-CM group vs the control group, *P < 0.05 ; LPS group vs CM + LPS group, ${ }^{*} \mathrm{P}<0.05$ ). All experiments were done in triplicate and repeated three times independently.

\section{Supplementary Files}

This is a list of supplementary files associated with this preprint. Click to download.

- S1.jpg 